\title{
Metastasis of Primary Cutaneous Adenoid Cystic Carcinoma to the Nasal Septum after 17 years: A Case Report
}

\author{
Yohei Takada ${ }^{a}$ Tomoko Takadab Sachie Takayasu ${ }^{b}$ Yuhei Ogino ${ }^{a}$ \\ Yuka Higashiyama $^{a}$ Kenta Fukui ${ }^{a}$ Daiki Sakamoto ${ }^{a}$ Mikiya Asako ${ }^{a}$ \\ Hiroshi Iwaic \\ aDepartment of Otolaryngology-Head and Neck Surgery, Kansai Medical University \\ Hospital Medical Center, Osaka, Japan; ${ }^{b}$ Department of Otolaryngology, JCHO Hoshigaoka \\ Medical Center Hospital, Osaka, Japan; 'Department of Otolaryngology-Head and Neck \\ Surgery, Kansai Medical University Hospital, Osaka, Japan
}

\section{Keywords}

Adenoid cystic carcinoma - Primary cutaneous adenoid cystic carcinoma - Nasal septal metastasis · Combined approach

\begin{abstract}
Primary cutaneous adenoid cystic carcinoma (PCACC) is extremely rare, and although distant metastasis has been reported, to date, there are no reports regarding metastasis to the nasal septum. We report a rare case of PCACC that metastasized to the nasal septum 17 years after the first surgery in a 59-year-old woman. She initially presented with a mass under the skin of her left mammary papilla. After a biopsy revealed the presence of an adenoid cystic carcinoma, the tumor was excised and definitively diagnosed as a PCACC. Five years after surgery, the patient presented with left lung metastasis and underwent a partial resection of the left lung. However, 8 years after this procedure, the patient had to undergo a partial resection of the right lung because of right lung metastasis. Four years thereafter, the patient presented with nasal septal metastasis. The tumor was excised successfully using a combined technique integrating intranasal and extranasal approaches. The patient is currently undergoing regular followup tests. Thus, in such cases, lifelong follow-up is necessary while checking for both distant metastasis and instances of local recurrence.
\end{abstract}

\section{Karger $\stackrel{\text { ! }}{=}$}




\section{Introduction}

Adenoid cystic carcinoma (ACC) is a malignant cancer that often develops in the head and neck region, especially the salivary glands. In addition, it can occur in the lacrimal glands, bronchi, ear canals, mammary glands, uterus, and various other organs; however, cases of primary cutaneous ACC (PCACC) are rare [1]. In this case, PCACC originated from the skin of the mammary papilla. Furthermore, the occurrence of lung and lymph nodes metastases is exceptionally rare in PCACC $[2,3]$. To the best of our knowledge, metastasis to the nasal septum has not been reported in the literature. We report a rare case of PCACC in a 59-year-old woman with two lung metastases and recurrence of metastasis to the nasal septum 17 years after the first surgery.

\section{Case Report}

\section{Past Medical History}

In 20XX, a 59-year-old woman presented with a mass under the left mammary papilla to the dermatology department, and a biopsy was performed. Histopathological findings revealed the presence of an ACC. Positron emission tomography-computed tomography (CT) was performed; however, no accumulation was observed in the head and neck region or the mammary gland, which are the most frequent sites of ACC. Therefore, PCACC was suspected, and the tumor and the left nipple were resected with a safety margin under general anesthesia. In addition, axillary lymph node dissection was performed. Histopathological findings showed that the tumor was localized to the skin, and a definitive diagnosis of PCACC was established based on the absence of mammary gland tissue.

In $20 \mathrm{XX}+5$, a nodular lesion was observed in the upper lobe of the patient's left lung on positron emission tomography-CT. A metastatic lung tumor was suspected, and a partial resection of the upper lobe of the left lung was performed under thoracoscopy. Histopathological findings showed an ACC similar to the PCACC, and distant metastasis from the skin was diagnosed.

In $20 X X+13$, the patient presented with a recurrence of the nodular lesion in the right lobe. Based on suspicion of metastatic lung tumor, partial resection of the upper lobe of the right lung was performed under thoracoscopy. The histopathological results were similar to those of the tests performed after the previous lung metastasis, confirming the presence of an ACC; therefore, a repeat diagnosis of distant metastasis from the skin was established.

\section{Current Medical History}

In $20 X X+17$, the patient noticed a left intranasal mass and was referred to our hospital for further assessment. Based on the findings of the nasal endoscopy performed during the initial consultation, we observed a mass on the left anterosuperior part of the nasal septum in the left nasal cavity (shown in Fig. 1a), and a biopsy was performed in the same site for diagnostic purposes. Based on the histopathological findings, ACC was diagnosed and considered a metastasis from the skin. The paranasal CT image (shown in Fig. 1b, c) revealed a soft shadow on the left anterosuperior side of the nasal septum, and the base of the tumor was observed to have extensively invaded the nasal septum. The boundary of a part of the septal cartilage was slightly obscured. Paranasal sinus magnetic resonance imaging (shown in Fig. 1d, e) showed low intensity on T1-weighted images and low-high intensity on T2-weighted images of the left anterosuperior side of the nasal septum, which was consistent with the CT findings. The tumor was confined to the nasal septum, and no evidence of infiltration to the nasal bone or skin was observed. Based on the imaging findings, we judged that the mass

\section{Karger'}




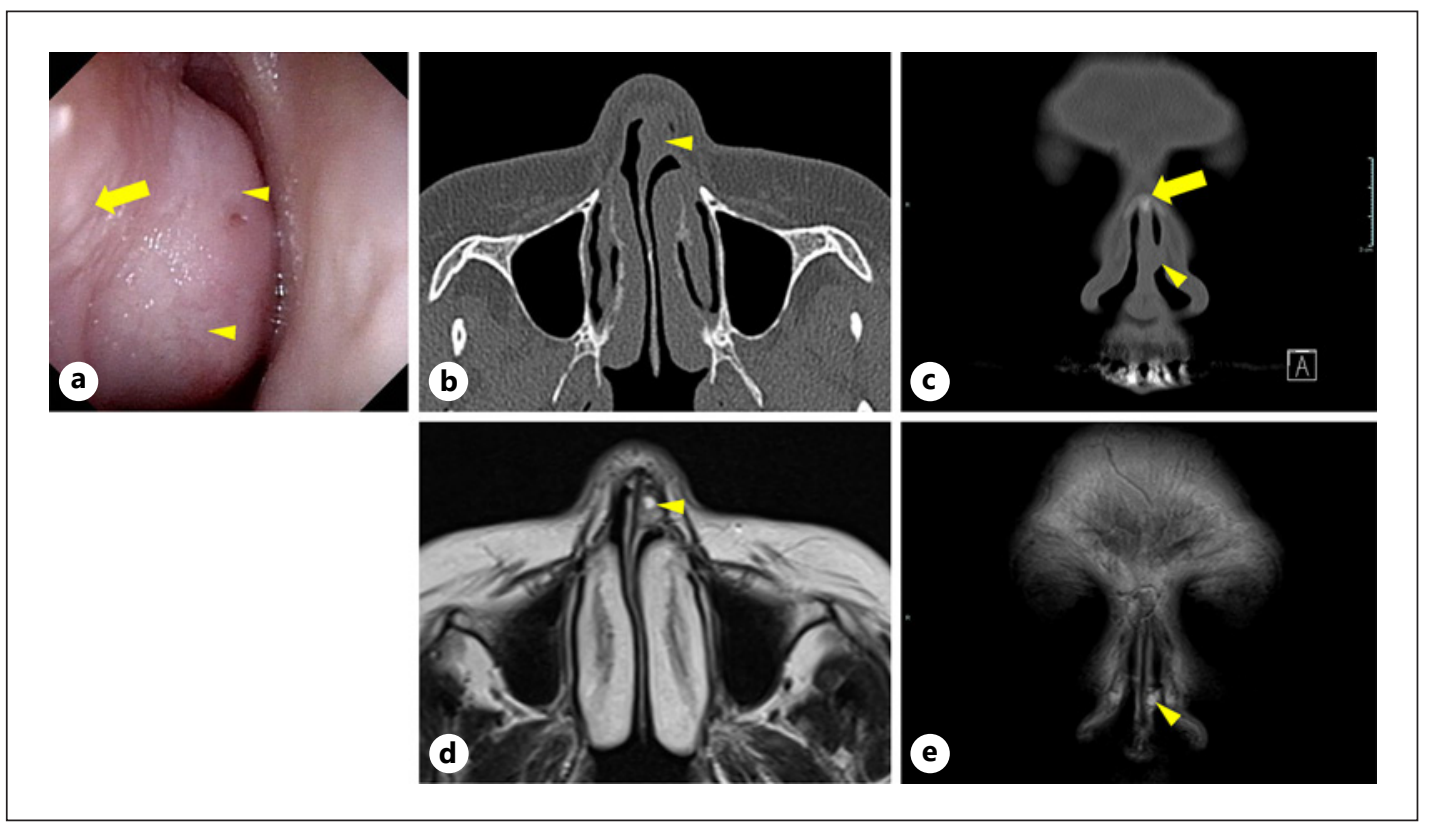

Fig. 1. Imaging examination. a An elevated lesion (arrowhead) with a broad base is observed on the left side of the nasal septum (arrow). Axial (b) and coronal (c) CT images show a soft tissue shadow on the left anterosuperior side of the nasal septum (arrowhead). The boundary with the nasal septal cartilage is unclear. No infiltration into the nasal bone (arrow) is observed. T2-weighted axial (d) and coronal (e) magnetic resonance images show a mixture of high and low signals in the left nasal cavity. On the left side (arrow) of the nasal septum, a mass with a mixture of low-intensity and high-intensity signals is observed in the anterosuperior region of the $\mathrm{T} 2$-weighted image.

could be resected with a portion of the nasal septal cartilage, and surgery under general anesthesia was planned. We believe that it was necessary to implement a combined approach integrating endoscopic sinus surgery and open septorhinoplasty to ensure a safety margin for excision from the tumor location.

\section{Surgery}

After careful observation of the interior of the nose and location of the base of the left nasal septal tumor, the safety margin for resection was set and marked, and an inverted $\mathrm{V}$-shaped incision line was created on the nasal columella (shown in Fig. 2a). Infiltration anesthesia was performed on the subperichondrium of the nasal septum, nasal columella, and back of the nose with $0.5 \%$ epinephrine. An incision was made with a scalpel using the extranasal method, the flap was raised to ensure no damage to the nasal septal cartilage, and the tip of the nasal septal cartilage was identified (shown in Fig. 2b). Subsequently, the operation was performed under an endoscope. The right nasal septal mucosa was incised with a scalpel and peeled in the anterior-posterior and superior-inferior directions under the nasal septal perichondrium to extensively expose the nasal septal cartilage (shown in Fig. 2c). No tumor was observed in the right nasal septal cartilage. Thereafter, the tumor in the left nasal septum was confirmed, and the anterosuperior mucosa was incised with an electric scalpel (shown in Fig. 2 d) along the determined safety margin line for excision. The same procedure was performed in the posterior and superior direction (shown in Fig. 2e), and the tumor was resected together with the nasal septal cartilage just below the tumor base (shown in Fig. 2f). The mucosae anterior, posterior, and superior to the tumor excision site were submitted for rapid histopathological diagnosis, and a negative margin was confirmed. The raised flap was 


\section{Case Reports in Oncology}
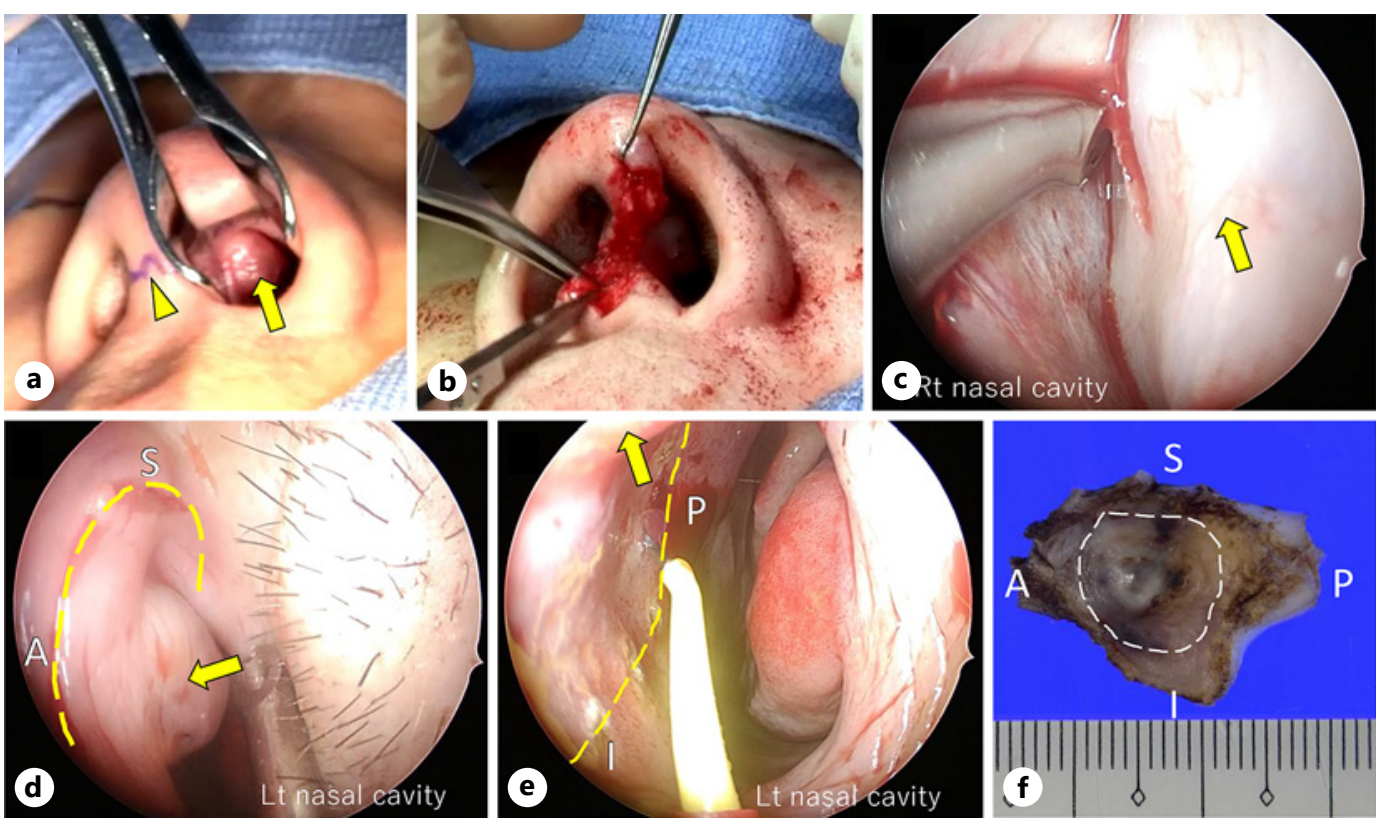

Fig. 2. Surgery. a After confirming the position of the left nasal septal tumor with a nasal speculum (arrow), an inverted V-shaped incision line (arrowhead) was created in the nasal column. b The flap was raised, and the tip of the septal cartilage was identified to ensure no damage to the septal cartilage. c The mucosa was peeled in the anterior and posterior direction and vertically in the subperichondrium of the right nasal septum to expose the nasal septal cartilage (arrow). d The location of the tumor (arrow) was confirmed, and a mucosal incision was made with anterior and superior excision margins (yellow dotted line). A: anterior, S: superior. e The location of the tumor (arrow) was confirmed, and a mucosal incision was made using an electric scalpel with an excision margin posteriorly and inferiorly (yellow dotted line). P: posterior, I: inferior. f The resected tumor (view from the left nasal surface). The part surrounded by the white dotted line is the tumor body. A: anterior, P: posterior, S: superior, I: inferior.

returned to its original position, and the surgery was completed by closing the skin with a 6-0 Prolene suture.

\section{Histopathological Findings}

ACC was diagnosed based on the mixed solid and cribriform patterns in the nasal septum using weak and enlarged hematoxylin/eosin (H/E) staining $(\times 1.25)$ (shown in Fig. 3a). Observation of the boundary between the tumor and the nasal septum cartilage using strong, enlarged $\mathrm{H} / \mathrm{E}$ staining $(\times 10)$ revealed that some tumors had invaded the nasal septal cartilage (shown in Fig. 3b). H/E staining $(\times 4)$ of the previously resected PCACC showed a tumor that formed large and small alveolar lesions from the dermis to the subcutaneous tissue, and ACC was diagnosed based on the mixed solid and cribriform patterns; notably, solid patterns were predominantly observed. In $\mathrm{H} / \mathrm{E}$ staining $(\times 4)$ of the metastatic lung tumor, a predominantly solid ACC with a mixed cribriform pattern was observed. Based on the clinical course and histopathological features, lung and nasal septum metastases from PCACC were diagnosed.

The postoperative course was uneventful, and the patient was discharged 3 days postoperatively. Postoperative chemoradiotherapy was not performed after consulting dermatologist and radiologist. One month after operation, nose endoscopy findings showed residual perforation of the nasal septum at the excised portion; however, no significant recurrence was observed 1 year and 8 months after operation. The patient is still undergoing follow-up. 


\section{Case Reports in Oncology}

\begin{tabular}{l|l}
\hline Case Rep Oncol 2022;15:99-105 \\
\hline DOI: 10.1159/000521978 & $\begin{array}{l}\text { ○ 2022 The Author(s). Published by S. Karger AG, Basel } \\
\text { www.karger.com/cro }\end{array}$ \\
\hline
\end{tabular}

Takada et al.: Nasal Septal Metastasis from Primary Cutaneous Adenoid Cystic Carcinoma
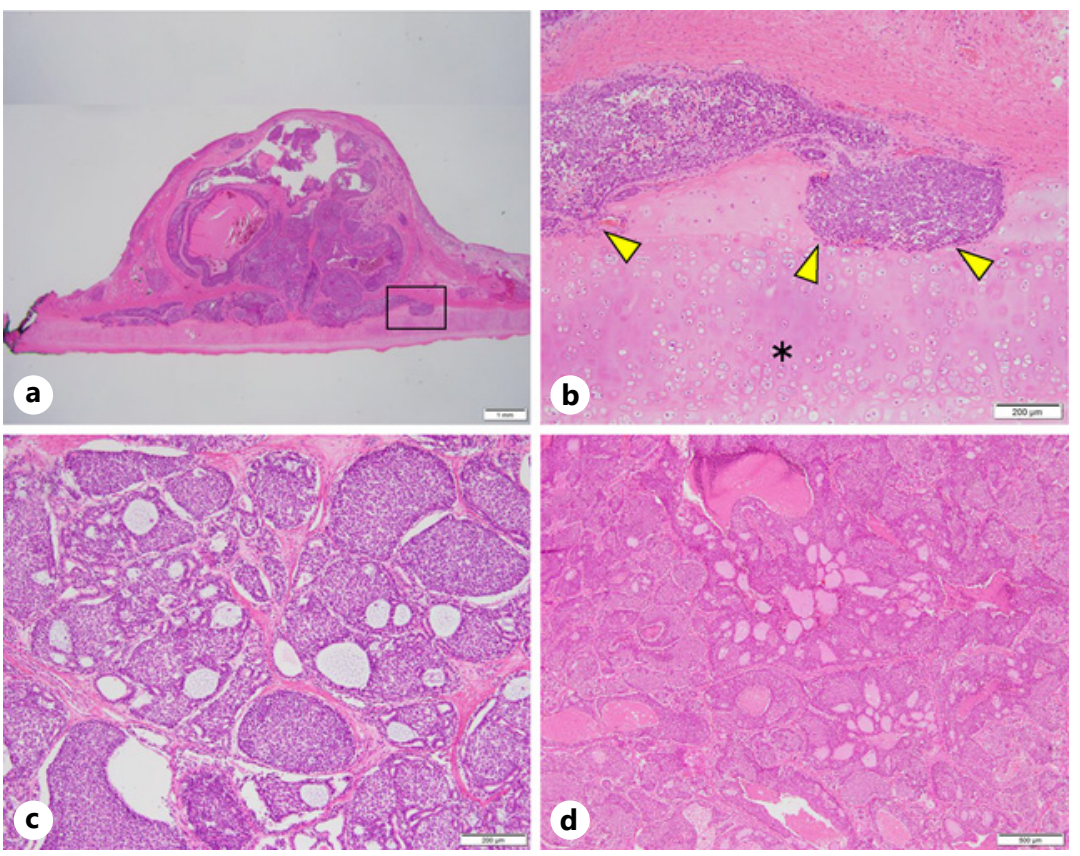

Fig. 3. Postoperative pathology studies. a Septal tumor: Weak and enlarged H/E staining ( $\times 1.25)$ : ACC with a mixt of solid and cribriform patterns in the nasal septum. $\mathbf{b}$ A strong enlargement of the nasal septal tumor with H/E stains ( $\times 10$ ): Enlarged view of the area enclosed by the square in Figure 1a. The tumor (arrowhead) has invaded the nasal septal cartilage $(*)$. c Papillary subcutaneous tumor H/E staining $(\times 4)$ : A tumor forming large and small cysts from the dermis to the subcutaneous tissue is observed and was diagnosed as ACC based on mixed solid and cribriform patterns. Solid patterns are predominant. $\mathbf{d}$ Lung tumor H/E staining $(\times 4)$ : ACC with a mixt of solid and cribriform patterns is observed in the lung. Solid patterns are predominant.

\section{Discussion}

We report a rare case of PCACC, which metastasized to the nasal septum 17 years after the first surgery. PCACC was first reported as a skin appendageal tumor by Boggio [4] in 1975; PCACC occurs most often in the head and neck, followed by the upper limbs, trunk, and lower limbs; however, it can occur in any part of the body [2].

According to a previous report, perineural invasion is an important feature noted in only $55 \%$ of cases [3]. PCACC shows a less aggressive behavior than the analogous tumor of the salivary glands [5]. The local recurrence rate of PCACC is 45\% [3], and the occurrence of lung and lymph nodes metastasis is exceptionally rare in PCACC $[2,3]$. There are reports of metastasis of PCACC to the lungs and brain, among other organs; however, cases of metastasis to the nasal septum have not been reported [2, 3, 6-10]. Therefore, we believe that the present case of metastasis of PCACC to the nasal septum is extremely rare.

Although the prognostic factors for local recurrence and metastasis are unclear for ACC, in 1978, Perzin et al. [11] classified ACC into three types based on patterns observed in histopathologic studies as follows: cribriform, tubular, and solid. The prognosis differs depending on the type, and it is reported that the solid type has a particularly poor prognosis.

In 1984, Szanto et al. [1] histologically classified ACC of salivary glands into three grades, with those consisting of luminal and cribriform patterns classified as Grade 1, those predominantly consisting of cribriform partially solid patterns (solid patterns constituting $<30 \%$ of the tumor area) as Grade 2, and those predominantly comprising solid patterns (solid patterns constituting $>30 \%$ of the tumor area) as Grade 3 . 
Tumors in which solid patterns comprise $\geq 30 \%$ of the area are associated with a poor prognosis $[1,11]$. In this case, histopathological examination at the first operation showed that the tumor predominantly comprised solid components (>30\%) and was, thus, categorized as Grade 3 according to Szanto's classification. Therefore, Grade 3 PCACC may have metastasized to the lungs and nasal septum due to the poor prognosis pattern.

The recommended treatment of PCACC is extensive surgical excision, with at least $2 \mathrm{~cm}$ of safety margin around the tumor to exclude frequent recurrence [12]. However, a simple excision results in more frequent recurrences; the local recurrence rate of PCACC is more than 50\% [12]. Although there are reports confirming the therapeutic effects of radiation therapy [13] and chemotherapy [14] as postoperative treatments, findings on chemotherapy and radiation therapy for radically unresectable cases have not been sufficiently accumulated.

Radiation therapy and chemotherapy should be administered to patients in whom resection is difficult or who may have tumors on the margin. In this case, the tumor could be removed by the combined approach of the intranasal and extranasal methods, and the surgical margin was negative; therefore, sufficient resection was considered possible. Regarding additional postoperative treatment, the surgical margin was negative, and distant metastasis from cutaneous ACC was observed; therefore, additional postoperative treatment was not performed after consulting a dermatologist and a radiologist. However, if local recurrence is observed in the nasal septum in the future, additional treatment may have to be considered; therefore, the patient is still undergoing follow-up.

PCACC requires long-term follow-up because metastatic lesions have been reported to occur more than 10 years after surgery [8]. However, in this case, metastasis was observed in the nasal septum 17 years after the first operation, with two lung metastases occurring in the intervening period. Therefore, we believe that lifelong follow-up is necessary while paying close attention to local recurrence and distant metastasis. Moreover, it is necessary to record a detailed medical history of patients with chronic PCACC, considering metastatic tumors in cases of intranasal ACC.

We believe that lifelong follow-up is necessary while paying close attention to local recurrence and distant metastasis. Moreover, it is necessary to record a detailed medical history of patients with chronic PCACC, considering metastatic tumors, in cases of intranasal ACC.

\section{Conclusion}

PCACC is a cancer with a high local recurrence rate, requiring long-term follow-up. We report a rare case of nasal septal metastasis occurring 17 years after the excision of a mammary papilla for PCACC. The nasal septal tumor including the nasal septal cartilage was completely excised through a combined approach of intranasal and extranasal methods. Notably, no recurrence has been observed to date.

\section{Acknowledgment}

We would like to thank Editage (www.editage.com) for English language editing.

\section{Statement of Ethics}

Written informed consent was obtained from the patient for the publication of this case report and any accompanying images. This retrospective review of patient data did not require ethical approval in accordance with local guidelines.

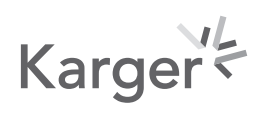




\section{Case Reports in Oncology}

\begin{tabular}{l|l}
\hline Case Rep Oncol 2022;15:99-105 \\
\hline DOI: 10.1159/000521978 & $\begin{array}{l}\text { @ 2022 The Author(s). Published by S. Karger AG, Basel } \\
\text { www.karger.com/cro }\end{array}$ \\
\hline
\end{tabular}

Takada et al.: Nasal Septal Metastasis from Primary Cutaneous Adenoid Cystic Carcinoma

\section{Conflict of Interest Statement}

The authors have no conflicts of interest to declare.

\section{Funding Sources}

The authors received no financial support for the research, authorship, and publication of this article.

\section{Author Contributions}

Y.T. and T.T. researched the literature and drafted the manuscript. Y.T and M.A. performed the surgeries. S.T. and H.I. critically revised the manuscript. Y.O., Y.H., K.F., and D.S. performed the examinations before and after surgery, provided photographs, and drafted the first version of the manuscript. All authors approved the final manuscript.

\section{Data Availability Statement}

All data generated or analyzed during this study are included in this article. Further enquiries can be directed to the corresponding author.

\section{References}

1 Szanto PA, Luna MA, Tortoledo ME, White RA. Histologic grading of adenoid cystic carcinoma of the salivary glands Cancer. 1984;54(6):1062-9.

2 Ramakrishnan R, Chaudhry IH, Ramdial P, Lazar AJ, McMenamin ME, Kazakov D, et al. Primary cutaneous adenoid cystic carcinoma: a clinicopathologic and immunohistochemical Study of 27 cases. Am J Surg Pathol. 2013;37(10):1603-11.

3 Prieto-Granada CN, Zhang L, Antonescu CR, Henneberry JM, Messina JL. Primary cutaneous adenoid cystic carcinoma with MYB aberrations: report of three cases and comprehensive review of the literature. J Cutan Pathol. 2017;44(2):201-9.

4 Letter BR. Adenoid cystic carcinoma of scalp. Arch Dermatol. 1975;111(6):793-4.

5 Wick MR, Swanson PE. Primary adenoid cystic carcinoma of the skin. A clinical, histological, and immunocytochemical comparison with adenoid cystic carcinoma of salivary glands and adenoid basal cell carcinoma. Am J Dermatopathol. 1986;8(1):2-13.

6 Seab JA, Graham JH. Primary cutaneous adenoid cystic carcinoma. J Am Acad Dermatol. 1987;17(1):113-8.

7 Sanderson KV, Batten JC. Adenoid cystic carcinoma of the scalp with pulmonary metastasis. Proc R Soc Med. 1975;68(10):649-50.

8 Zimmerman RL, Bibbo M. Fine needle aspiration diagnosis of a pulmonary metastasis from a cutaneous adenoid cystic carcinoma. A case report. Acta Cytol. 1998;42(2):367-70.

9 Chang SE, Ahn SJ, Choi JH, Sung KJ, Moon KC, Koh JK. Primary adenoid cystic carcinoma of skin with lung metastasis. J Am Acad Dermatol. 1999;40(4):640-2.

10 Pozzobon LD, Glikstein R, Laurie SA, Hanagandi P, Michaud J, Purgina B, et al. Primary cutaneous adenoid cystic carcinoma with brain metastases: case report and literature review. J Cutan Pathol. 2016;43(2):137-41.

11 Perzin KH, Gullane P, Clairmont AC. Adenoid cystic carcinomas arising in salivary glands: a correlation of histologic features and clinical course. Cancer. 1978;42(1):265-82.

12 Van der Kwast TH, Vuzevski VD, Ramaekers F, Bousema MT, Van Joost T. Primary cutaneous adenoid cystic carcinoma: case report, immunohistochemistry, and review of the literature. Br J Dermatol. 1988;118(4):567-77.

13 Vikram B, Strong EW, Shah JP, Spiro RH. Radiation therapy in adenoid-cystic carcinoma. Int J Radiat Oncol Biol Phys. 1984;10(2):221-3.

14 Ikegawa S, Saida T, Obayashi H, Sasaki A, Esumi H, Ikeda S, et al. Cisplatin combination chemotherapy in squamous cell carcinoma and adenoid cystic carcinoma of the skin. J Dermatol. 1989;16(3):227-3. 